\title{
Managerial Forecasting System Based on RBF Neural Network for Financial Data
}

\author{
Dusan Marcek ${ }^{1,2}$ \\ ${ }^{1}$ Research Institute of the IT4Innovations Centre of Excellence, The Silesian University \\ Opava, Czech Republic \\ ${ }^{2}$ The Faculty of Management Science and Informatics University of Zilina, Slovakia
}

\begin{abstract}
Forecasting systems are applied which are based on the latest statistical theory and artificial neural networks. The impact of these methods to risk reduction is judged in managerial decision-making. The fundamental question arises whether non-linear methods like neural networks can help modeling any non-linearities being inherent within the estimated statistical model. The proposed novel modeling approach is applied to high frequency time series of EUR/USD exchange rates (EUR currency against the US dollar. Our results show that the proposed neural approach achieves better forecast accuracy on the validation dataset than most available statistical techniques.
\end{abstract}

Keywords: Forecasting models, neural networks, MDS (Managerial Decision Making, accuracy of forecasting models.

\section{Introduction}

An important sphere of information necessary for management of economic processes on all managerial levels is the information about the future development of quantities expressed quantitatively, which is used to characterize the state and the development of the object or process. Evidence shows that it is possible to reduce uncertainty by a suitable choice and use of forecasting models based on statistical methods, soft computing and artificial intelligence methods. In comparison with the manager's expert estimates, these models based on statistical and soft computing are capable of providing information in the form of forecasts of quantities with an acceptable degree of uncertainty. The manager using these forecasts is able to make better decisions, i.e. such decisions whose risks in achieving targets are minimized.

One of the approaches to understanding uncertainty in forecasting models is understanding it as the standard deviation $\sigma$ of the forecasted quantity used e.g. in , [1], [2] and so on. The standard deviation as a degree of uncertainty or risk of forecasted quantity values estimates is proportional to the statistical degree of accuracy of the forecast defined as Root Mean Square Error (RMSE) or MAPE (Mean Absolute Percentage Error). This approach is used with measuring risks of prognoses of many economic and financial forecasting models, and in forecasting models of economic time series, models for managing financial risk and so on.

The aim of the paper is to explain achieved aspects of both statistical and soft approaches for quantifying forecast accuracy applied to daily EUR/USD exchange rate time series and assess their prediction performance. The paper is organized as follows. In Section 2 we de- 
scribe the basic ARCH-GARCH model and its variants: EGARCH, PGARCH and TGARCH models. In Section 3 we present the data, conduct some preliminary analysis of the time series and demonstrate the forecasting abilities of ARMA (AutoRegressive Moving Average)ARCH/GARCH modes on an application. In Section 4 we introduce the architectures of RBF neural networks used in a prediction. Section 6 presents results our experimental study and puts an empirical comparison. Section 7 briefly concludes.

\section{Some ARCH-GARCH Models for Financial Data}

In this section we propose a linear ARCH-GARCH type model in order to forecast the EUR/USD-exchange rate. Our database is composed of 3652 observations (from January 2, 2001 to February 28,2011 ) of the average daily prices for EUR currency against the US dollar (denoted EUR/USD). Figure 1 graphs this time series (denoted as $y$ in USD).

As we would like to develop a time series model for one daily ahead forecast of the changes in the EUR/USDexchange, the sample period for analysis from January 2, 2001 to October 30, 2010, (denoted as dataset A or training data set) was defined, i.e. the period over which the forecasting model can be developed and the ex post forecast period from November 1, 2010 to December 31, 2010 (denoted as dataset $\mathrm{E}$ or validation or ex-post data set) as the time period from the first observation after the end of the sample period to the most recent observation.

Input selection is crucial importance to the successful development of an ARCH-GARCH model. Potential inputs were chosen based on traditional statistical analysis: these included the raw data series of the EUR/USD exchange rate and lags thereof.

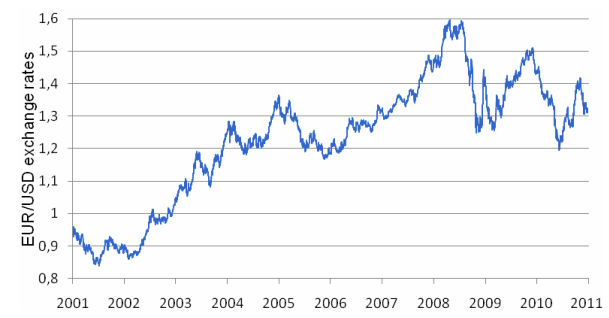

Fig. 1: Time series of daily exchange rate changes EUR currency against US dollar (2001-2011).

The relevant lag structure of potential inputs was analysed using traditional statistical tools, i.e. using the autocorrelation function (ACF), partial autocorrelation function (PACF) and the Akaike/Bayesian information criterion (AIC/BIC). We looked to determine the maximum lag for which the PACF coefficient was statistically significant and the lag given the minimum AIC. According to these criterions the specification and estimation was performed by means of the R2.6.0 software (available at http://cran.r-project.org) and resulted into best $\operatorname{ARIMA}(1,1,1)+\operatorname{PGARCH}(1,1)$ model in the form of mean and variance equations respectively as follows

$\Delta y_{t}=\xi+\phi_{1} \Delta y_{t-1}+\theta_{1} \varepsilon_{t-1}+\varepsilon_{t}$,
$h_{t}^{d}=\alpha_{0}+\sum_{i=1}^{p} \alpha_{i}\left(\left|\hat{\varepsilon}_{t-i}\right|+\gamma_{i} \hat{\varepsilon}_{t-i}\right)^{d}+\sum_{j=1}^{q} \beta_{j} h_{t-j}^{d}$

and fitted as

$\Delta y_{t}=0.000126+0.079563 \Delta y_{t-1}+0.041943_{1} \varepsilon_{t-1}+\varepsilon_{t}$,

$h_{t}^{1.836}=2.26 .10^{-7}+0.0303\left(\left|\hat{\varepsilon}_{t-1}\right|+0.00803 \hat{\varepsilon}_{t-1}\right)^{1.8365}$

$+0.96015 h_{t-1}^{1.836}$

where $d$ is a positive exponent, $\left\{\varepsilon_{t}\right\}$ is a sequence of iid random variables with zero mean and unit variance, $\alpha_{i}$ a $\beta_{i}$ are the $\mathrm{ARCH}$ and GARCH parameters, $h_{t}$ represent the conditional variance of time series and $\xi, \phi_{1}, \theta_{1}$ are unknown parame- 
ters of the ARIMA $(1,1,1)$ model. To test the adequacy of the developed model we applied the standardized residuals (3.4) which are calculated from the last two equations on the data set $\mathrm{A}$, to the BDS test. The BDS test confirmed presence of non-linearity in the standardized residuals. So, we can conclude that there is no evidence to reject the model. After these findings we can make predictions for next 54 trading days using the model with the smallest RMSE, i.e. by the $\operatorname{ARIMA}(1,1,1)$ + PGARCH(1,1) model with Gaussian error distribution. These predictions are calculated by means of the Eviews software and shoved in Figure 2. The statistical summary measures of model's forecast accuracy are given in Table 1.

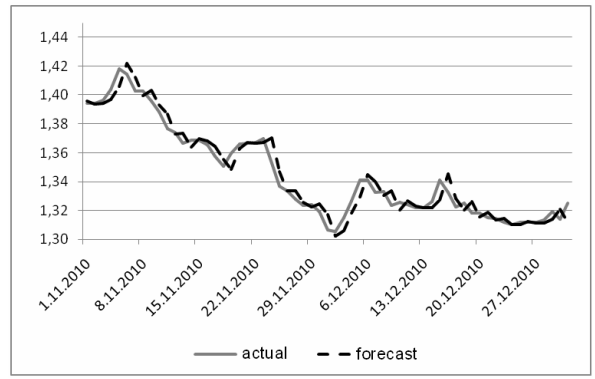

Fig. 2: Actual (solid) and forecast (dotted) values of the EUR/USD exchange rate.

Table 1: The statistical summary measures of model's forecast accuracy (statistical alternative).

\begin{tabular}{|l|c|c|c|}
\hline Measures & RMSE & MAE & MAPE \\
\hline $\begin{array}{l}\text { Models: } \\
(1)+(2)\end{array}$ & 0.00793 & 0.00646 & 0.00022 \\
\hline
\end{tabular}

\section{Neural Approach}

The same data used for estimating the linear $\operatorname{ARIMA}(1,1,1)+\operatorname{PGARCH}(1,1)$ model was also used to train neural network. The variables forming the right hand of the model given by Eq. (1) above were used as input units.

For the investigation of the neural networks a granular RBF (Radial Basic
Function) net was employed [Kec] (see Fig. 3), where the weights $v_{j}$ have been trained by using Backpropagotion. The transfer function in the hidden layer is a Gaussian RBF with cloud concept [3], whereas for the output unit a linear transfer function is applied. The output values $O_{j}^{N}$ from the hidden layer are "normalized", i. e. the values whose sum is equal to 1 . The weights $w_{j}$ are the centers $c_{j}$ of the radial basis functions [4]. To find the weights $\mathrm{w}_{j}$ or centers $\mathbf{c}_{j}$ of activation functions we used the adaptive (competitive learning) version of Kohonen's rule for clustering input data set.

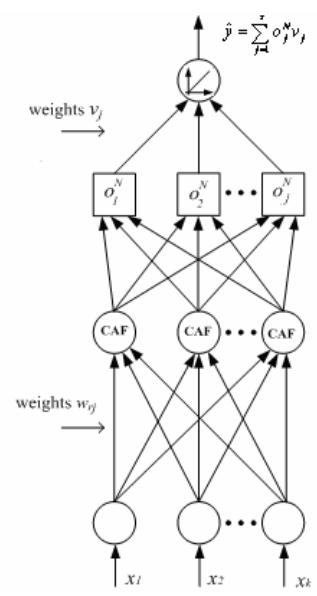

Fig. 3.: Granular RBF neural network architecture.

As the neural network in this study is used as a non-linear supplement of linear $\operatorname{ARIMA}(1,1,1)+\operatorname{PGARCH}(1,1)$ approach the network architecture allows linear input/output-links to be included. In order to avoid over-fitting and data-fitting, the network was kept simple (the number of hidden units were 10 , the learning rate was set to 0.05 . The ex post forecasts are shown in Fig. 4 and the corresponding results of the ex post forecasts evaluation by statistical summary measures of model's forecast accuracy are shown given in Table 2 . 


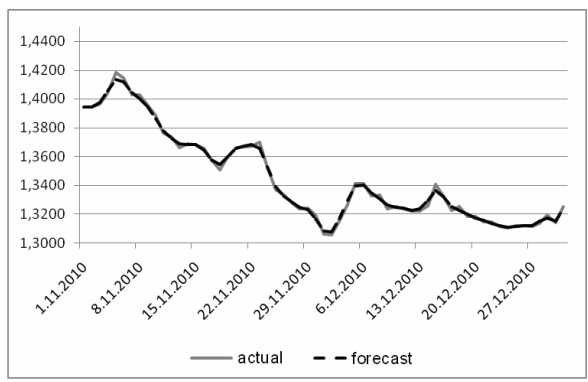

Fig. 4: Actual (solid) and forecast (dotted) values of the EUR/USD exchange rate forecast (Neural alternative of $\operatorname{ARIMA}(1,1,1)+$ PGARCH(1,1) model).

Table: 2: The statistical summary measures of model's forecast accuracy (neural alternative).

\begin{tabular}{|l|c|c|c|}
\hline Measures & RMSE & MAE & MAPE \\
\hline $\begin{array}{l}\text { Models: } \\
(1)+(2)\end{array}$ & 0.00185 & 0.00145 & 0.00107 \\
\hline
\end{tabular}

\section{Empirical Comparison}

From Tables' 1,2 it is shown that both forecasting models used are very accurate. The development of the error rates on the validation data set showed a high inherent deterministic relationship of the underlying variables. Though promising results have been achieved with both approaches, for the chaotic financial time markets a purely linear (statistical) approach for modeling relationships does not reflect the reality. For example, if investors do not react to a small change in exchange rate at the first instance, but after crossing a certain interval or threshold react, then a non-linear relationship between $\Delta y_{t}, \Delta y_{t-1}$ and $\varepsilon_{t-1}$ exists in model (1). The neural approach based on granular RBF network not only detected the functionality between the underlying variables and the EUR/USD exchange rates as well as the short-run dynamics. Moreover the RBF neural network has such attributes as computational efficiency, simplicity, and ease adjusting to changes in the process being forecast. ARCH-GARCH models require more costs of development, installation and operation in a management system, management comprehension and co-operation, and often a lot of computational time. A serious drawback of ARIMA-GARCH models is the investment in time and other resources required to build a satisfactory model.

\section{Conclusion}

Overall, the study indicates that there is a great deal of linearity to be extracted from the data. Our study also proved that it is possible to achieve significant risk reduction in managerial decision-making by applying modern forecasting models based on information technology such as neural networks developed within artificial intelligence.

Acknowledgments. This work was supported by the European Regional Development Fund in the IT4Innovations Centre of Excellence project (CZ.1.05/1.1.00/02.0070).

\section{References}

[1] M. MARCEK, M. "Statistical and RBF NN models: Providing forecast and risk assessment," Central European Review of Economic Issues 12:, 175-82, 2009.

[2] D. Applebaum D. "Lévy Processes and Stochastic Calculus," (Cambridge: Cambridge University Press, 2004).

[3] D. LI, and Y. DU. "Artificial intelligence with uncertainty," Boca Raton: Chapman \& Hall/CRC, Taylor \& Francis Group, 2008.

[4] V. Kecman, "Learning and soft computing: support vector machines, neural networks, and fuzzy logic," (Massachusetts: The MIT Press, 2001). 